\title{
Discussion on the Local Magnetic Force between Reversely Magnetized Micro Metal Particles in the Microwave Sintering Process
}

\author{
Yu Xiao, Feng Xu *, Bo Dong, Wenchao Liu and Xiaofang $\mathrm{Hu}$ \\ CAS Key Laboratory of Mechanical Behavior and Design of Materials, Department of Modern Mechanics, \\ University of Science and Technology of China, Hefei 230026, China; xiaoyuxy@mail.ustc.edu.cn (Y.X.); \\ dongbo@mail.ustc.edu.cn (B.D.); liuwc@mail.ustc.edu.cn (W.L.); huxf@ustc.edu.cn (X.H.) \\ * Correspondence: xufeng3@ustc.edu.cn; Tel.: +86-551-6360-0564; Fax: +86-551-6360-6459 \\ Academic Editor: Hugo F. Lopez \\ Received: 4 December 2016; Accepted: 1 February 2017; Published: 8 February 2017
}

\begin{abstract}
Synchrotron radiation computed tomography was applied to investigate $\mathrm{Cu}-\mathrm{Fe}$ mixture microwave sintering in situ and to examine the magnetic force between reversely magnetized micro-metal particles in microwave sintering. Results revealed that the growth rate of the sintering necks between $\mathrm{Cu}-\mathrm{Fe}$ particles and $\mathrm{Cu}-\mathrm{Cu}$ particles around the iron particles distributed in a vertical direction was faster than that of the sintering necks in the horizontal direction. These phenomena were consistent with the possible influence caused by the magnetic force between metal particles, as shown in our simple particle model. The kinetic curves of sintering neck growth along the vertical and horizontal directions quantitatively revealed the difference in growth rates. The contributing factors of magnetic force in microwave sintering were subsequently discussed. The volume of iron particles was proportional to the influence of magnetic force, and their shape elicited a remarkable influence based on demagnetization effects. This study provided a useful basis for microwave sintering mechanisms and anisotropic material preparation.
\end{abstract}

Keywords: microwave sintering; microstructure; synchrotron radiation computed tomography

\section{Introduction}

Microwave sintering is a novel material preparation method [1-4] preferred over conventional sintering because of special advantages, including high heating rate, overall and even heating, and material microstructure improvement. Roy et al. [5] confirmed that the modulus of $\mathrm{Fe}-\mathrm{Ni}$ rupture is $60 \%$ higher than that of conventional specimens after 10-30 min of microwave treatment. The excellent advantages of microwave sintering are attributed to the unique driving force caused by microwave electromagnetic field [6,7]. By comparison, conventional sintering is stimulated by thermal field. Electromagnetic field elicits various non-thermal effects except microwave thermal effect. Janney et al. [8] indicated that the activation energy is lower in microwave sintering than in conventional sintering. Electromagnetic field also influences mass diffusion during sintering $[9,10]$. Other theories, including crystallization enhancement [11] and polarization effects [12], have been proposed. The effects of microwave electromagnetic-induced force should also be considered [13-15]. Although studies have been performed on the effects of force on microwave sintering for ceramics, the effects of force on microwave sintering for metals, first developed in 1999 [5], have yet to be fully described.

Metals are magnetized within a magnetic field, and a magnetic force exists between magnetized objects. We aim to determine whether a force exists and influences microwave sintering for metals with a micro particle size that is less than the wavelength of a microwave field. However, experiments are 
not easily performed through conventional techniques because of complex and extreme environments characterized by electromagnetic radiation and high temperature in microwave sintering. Fortunately, microstructure evolution during microwave sintering can be directly observed through synchrotron radiation computed tomography (SR-CT). SR-CT is a novel testing technology that can achieve non-destructive and real-time 3D observations in extreme environments, such as high or low temperature, high pressure, and intense radiation. In sintering studies $[16,17]$, SR-CT can continuously obtain accurate experimental data regarding surface and internal 3D microstructure evolution without interrupting the process and destroying the samples. Quantitative analysis can then be conducted on the basis of SR-CT experimental data.

Copper and iron exhibit different magnetic properties. $\mathrm{Cu}-\mathrm{Fe}$ particle microwave sintering was conducted to examine the magnetic force between their reversely magnetized micro metal particles. Microstructure evolution occurring inside the particles during sintering was observed in situ through SR-CT. The influence of the local magnetic force between heterogeneous metal particles was discussed on the basis of images showing microstructure evolution. A model based on the magnetic force between magnetized particles was also proposed to predict the possible phenomenon. Local microstructure evolution processes and sintering neck growth curves were determined. Our results revealed that sintering necks around iron particles exhibited different growth rates at various directions. Our experimental results were also consistent with the model prediction. Further observations in some local regions with different iron particles showed that the volume of particles influences magnetic force, and this finding was similar to those shown in macro objects. This work verified the influence of magnetic force between reversely magnetized micro-metal particles in microwave sintering. This work further provided insights into metal microwave sintering mechanisms and proposed possible approaches to produce anisotropic materials.

\section{Experiment and Results}

A microwave sintering experiment was conducted on the BL13W1 beam line at the Shanghai Synchrotron Radiation Facility (SSRF, Shanghai, China, a third-generation light source). The CCD resolution was $0.33 \mu \mathrm{m}$ per pixel. Pure copper powder and iron powder with an average diameter of approximately $70 \mu \mathrm{m}$ were used. Copper powder and iron powder (+170 to 270 mesh and -200 mesh, respectively, from Alfa Aesar, Shanghai, China.) were mixed at volume ratios of $85 \%$ copper and $15 \%$ iron by ball milling and then loosely poured into a quartz capillary with an inner diameter of approximately $0.3 \mathrm{~mm}$ and a height of approximately $10 \mathrm{~mm}$. The initial relative density calculated on the basis of SR-CT data was 49\%. Some copper particles were fixed on the outer capillary surface as marking points to easily locate the same region at different sintering times. A SiC susceptor was used to preserve heat and to accelerate the increase in sample temperature because of the small sample size and large space of the cavity chamber. The sample was subsequently introduced into a single-model microwave cavity with an output power of $0-1.5 \mathrm{~kW}$. The size of the resonator was $222 \mathrm{~mm}$ (length) $\times 109 \mathrm{~mm}$ (width) $\times 54 \mathrm{~mm}$ (height). The microwave electromagnetic field type can be adjusted by using a short piston. The experimental system is shown in Figure 1.

The sample was set at the position with the maximum magnetic field. Temperature was measured using an infrared thermometer. Nitrogen was used to create a protective atmosphere and avoid oxidation. Projections were captured every $1.5^{\circ}$ during the $180^{\circ}$ or sample rotation. The X-ray energy was $38 \mathrm{Kev}$ and the exposure time was $500 \mathrm{~ms}$. The temperature was measured by a high-accuracy mono infrared thermometer $\left( \pm 0.3 \%\right.$ accuracy, $250{ }^{\circ} \mathrm{C}-1800{ }^{\circ} \mathrm{C}, 0.9$ emissivity; CTlaser 3M, Optris, Berlin, Germany). The curves of temperature and microwave power are shown in Figure 2. The temperature was maintained below the Curie temperature of iron $\left(770^{\circ} \mathrm{C}\right)$ for several CT series and then increased to $800{ }^{\circ} \mathrm{C}$ by adjusting the microwave power. 


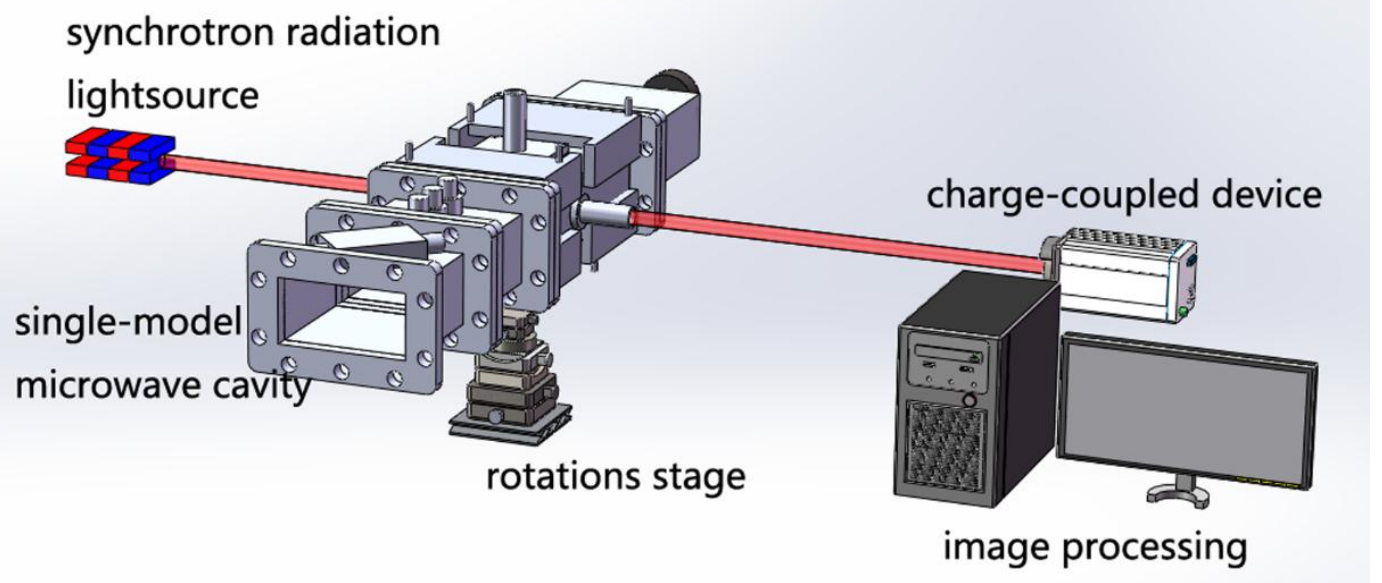

Figure 1. Schematic of in situ synchrotron radiation computed tomography (SR-CT) system of microwave sintering.

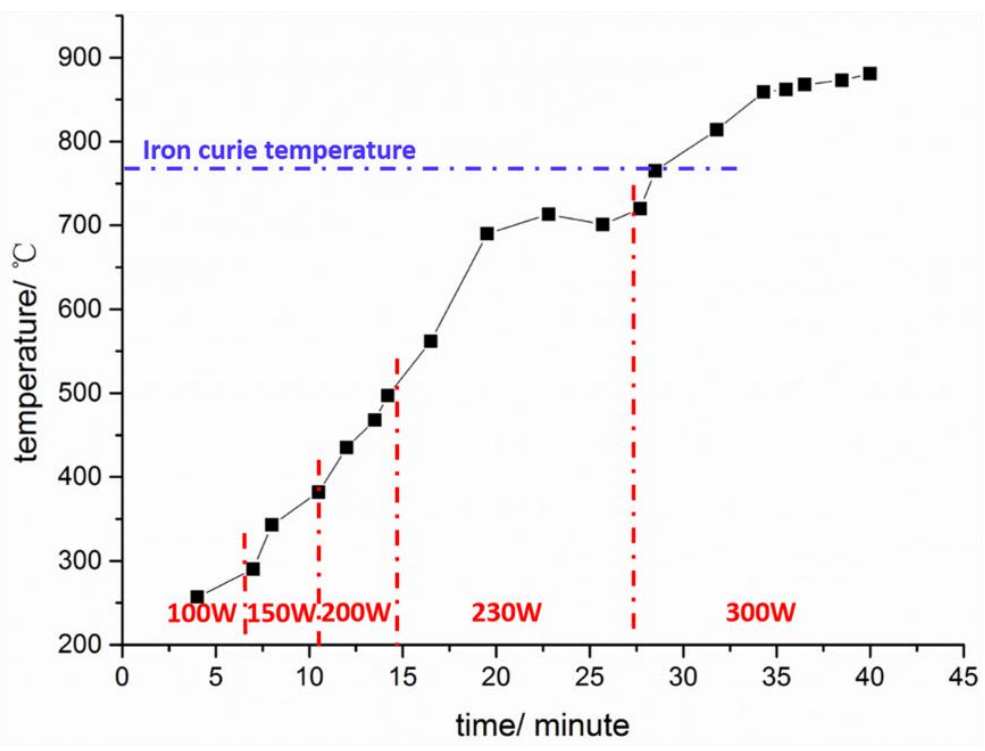

Figure 2. Curve of temperature and microwave power.

The CT images were reconstructed using the Algebraic Filtered-Back-Projection and Total Variation Minimization (AFBP-TVM) sparse algorithm [18]. The slices were stacked into 3D images and visualized in Volume Graphics (VG). The three-dimensional microstructure evolution process obtained by SR-CT is shown in Figure 3. Sintering phenomena including pore shrinkage, sintering neck growth and sample densification were observed. Our discussions were based on two-dimensional slices to describe the inside microstructure. 


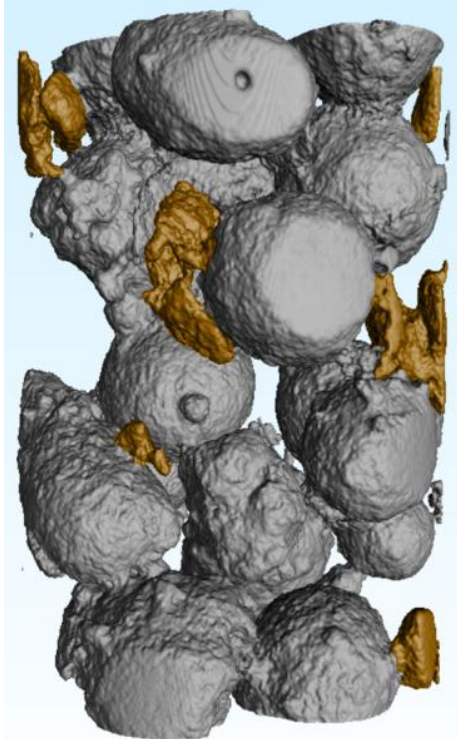

$\mathrm{T}=5 \mathrm{~min}$

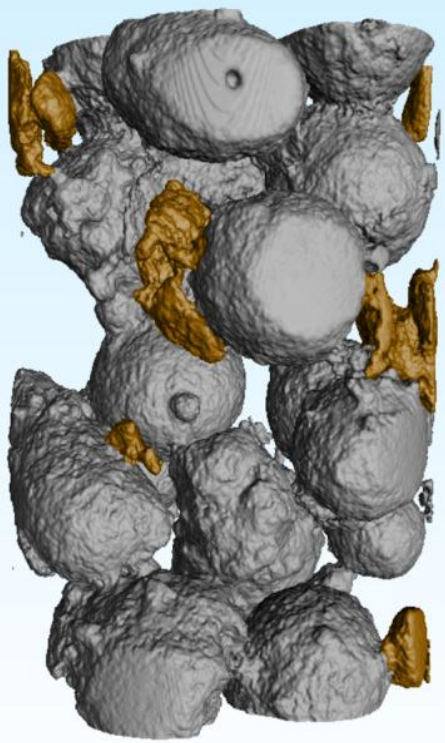

$\mathrm{T}=17 \mathrm{~min}$

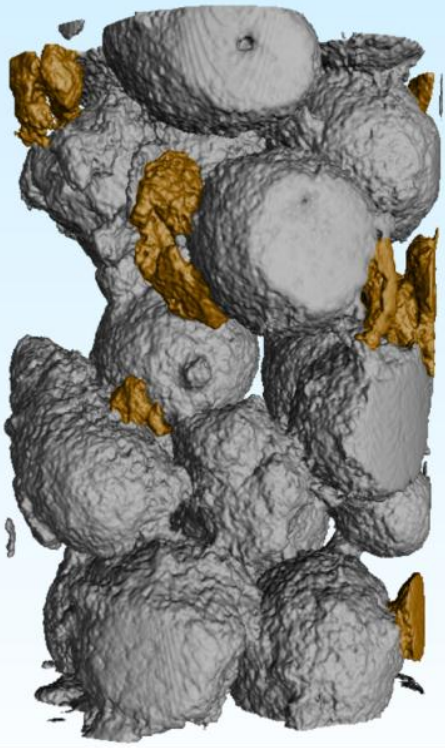

$\mathrm{T}=27 \mathrm{~min}$

Figure 3. 3D images of the microstructure at different sintering times.

\section{Discussion}

In this section, the magnetic force between micro particles in microwave sintering process was discussed on the basis of actual experiment results. A simple model was used to speculate possible phenomena, and corresponding phenomena were extracted from the CT images to verify the mode. The influencing parameters of the magnetic force effect were described on the basis of direct experimental phenomena and quantitative statistical analysis.

\subsection{Discussion on the Influence of Magnetic Force between Reversely Magnetized Micro Metal Particles in Microwave Sintering}

If the magnetic force between micro metal particles affects microwave sintering, special sintering phenomena compared with conventional sintering likely occurred. The magnetic susceptibility of iron is significantly larger than that of copper. Hence, we focused on the regions around the iron particles in the sample. Coupled particles of $\mathrm{Cu}-\mathrm{Fe}$ and $\mathrm{Cu}-\mathrm{Cu}-\mathrm{Fe}$ are produced after iron particles are introduced, and two-dimensional images of the regions containing these coupled particles are extracted and shown in the following.

A simple model comprising $\mathrm{Cu}-\mathrm{Fe}$ coupled particles is proposed to speculate possible phenomena. Iron is ferromagnetic and copper is diamagnetic and the magnetization direction of the two particles is opposite from each other. The magnetic force between them is further discussed in two typical relative positions of the particles and magnetic field. When the magnetic field direction is perpendicular with the line of two particles' centers, attractive force exists as shown in Figure 4a. When the magnetic field direction is parallel with the line of two particle centers, the magnetic force would be repulsive as Figure $4 \mathrm{~b}$ shows. However, the magnitude of magnetic force varies inversely with the square of the distance, represented by D in Figure 4, hence the repulsive force is stronger than the attractive force. Although both attractive and repulsive forces are quite small because of the magnetic susceptibility of copper, the function of this force on a micro-scale is yet to be determined. According to this model, the sintering neck growth around iron particles might be influenced by the magnetic force. 


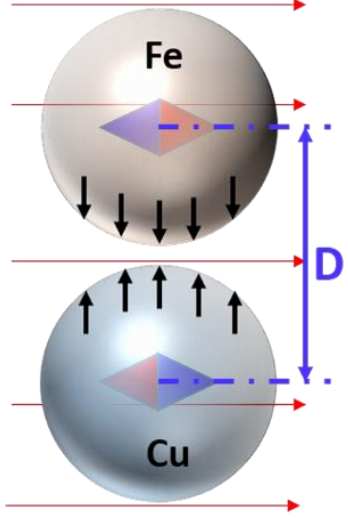

(a) Attractive force

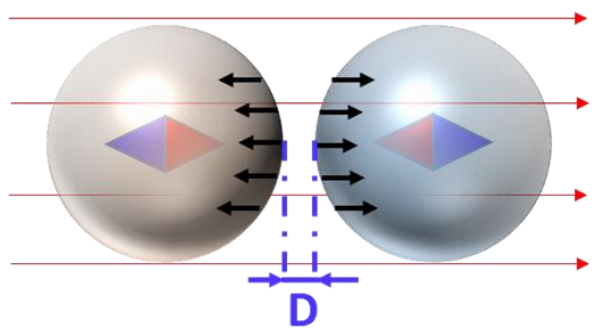

(b) Repulsive force

Figure 4. Schematic of magnetic force between particles. The magnetic field direction is perpendicular (a) and parallel (b) with the line of two particles' centers.

The position of the sample in the microwave field during actual sintering is shown in Figure 5. The sample was placed in the region with the maximum magnetic field by adjusting the short piston. The local microwave magnetic field around several particles could be regarded as uniform because particle size was smaller than the microwave wavelength. As the sample rotated when the CT images were captured, the relative direction between the magnetic field and particles changed. This observation differed among particles arranged in vertical and horizontal directions.
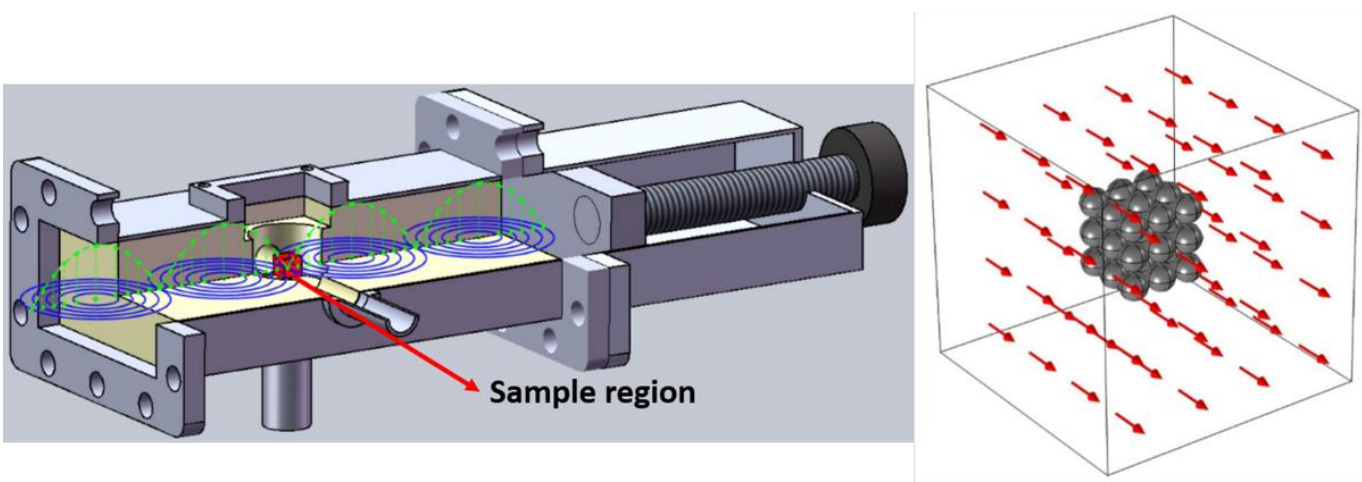

Figure 5. Sample position in the microwave magnetic field.

The position of the microwave magnetic field and particles was similar to that shown in Figure 4a when $\mathrm{Cu}-\mathrm{Fe}$ coupled particles were arranged in the vertical direction. An attractive force occurred between $\mathrm{Cu}-\mathrm{Fe}$ particles, and the sintering neck growth rate was improved. Conversely, the relative position of the microwave magnetic field and particles was altered when $\mathrm{Cu}-\mathrm{Fe}$ coupled particles were arranged in the horizontal direction because the sample rotated when the CT images were captured. A repulsive force occurred when the position was similar to that in Figure 4a, whereas an attractive force was observed when the position was similar to that illustrated in Figure $4 \mathrm{~b}$. The repulsive force was stronger than the attractive force because of the difference in the distance between the equivalent magnetic poles of the particles. Hence, the sintering neck between $\mathrm{Cu}-\mathrm{Fe}$ particles arranged in the horizontal direction was restrained as a whole.

Some local regions in the sample containing $\mathrm{Cu}-\mathrm{Fe}$ coupled particles are extracted and depicted in the next figures. In Figure 6, several spherical copper particles surround an irregular iron particle. According to analysis, the sintering neck growth in the vertical direction would improve while the sintering neck growth in the horizontal direction would be restrained. As Figure 6 shows, the initial 
distance between the irregular iron particle in the rectangle and the spherical copper particle above is large, but the sintering neck grew obviously large. On the contrary, no neck was found between iron and copper particles on both sides, although the initial distance between these particles was short. From the microstructure evolution, iron particles would improve the sintering process in the vertical direction and restrain it in the horizontal direction because of the magnetic force between $\mathrm{Cu}-\mathrm{Fe}$ coupled particles.

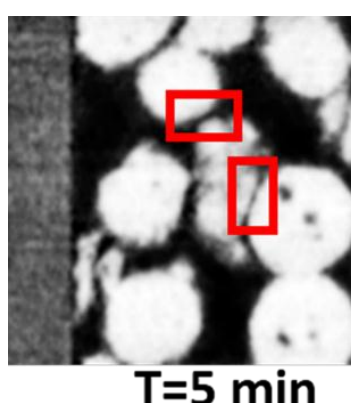

(a)

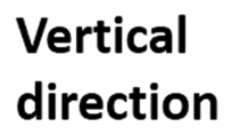

(b) Horizontal direction

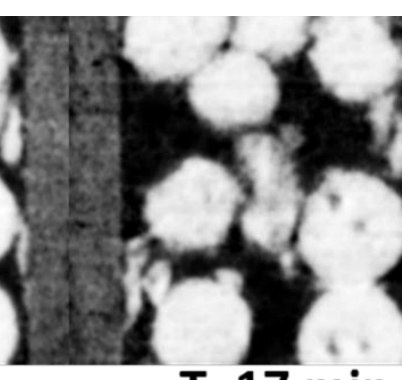

$\mathrm{T}=17 \mathrm{~min}$
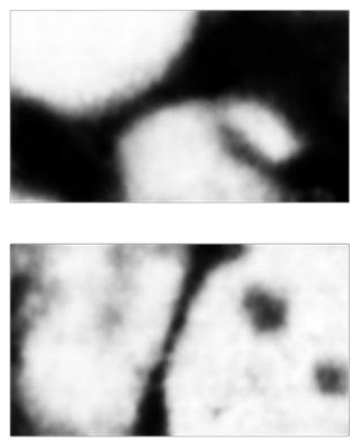

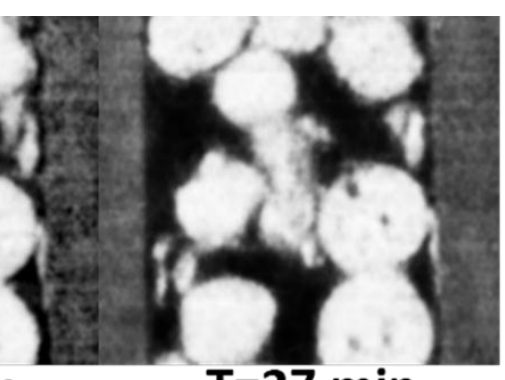

$\mathbf{T}=\mathbf{2 7} \mathrm{min}$
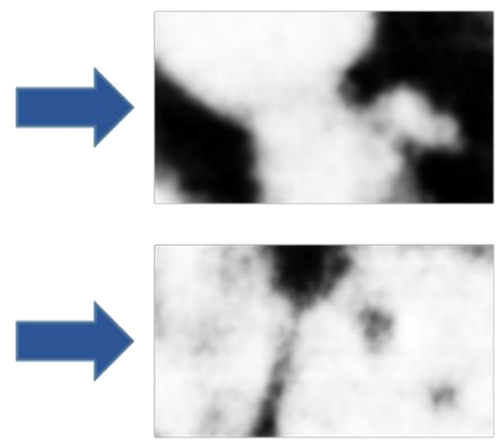

Figure 6. Local microstructure evolution of $\mathrm{Cu}-\mathrm{Fe}$ necks in the vertical direction at different sintering times. Enlarged view of the coupled particles in vertical direction (a) and horizontal direction (b).

Furthermore, the influence of the magnetic force on $\mathrm{Cu}-\mathrm{Cu}$ necks around iron particles can also be easily inferred based on the model above. When the coupled particles are arranged in a vertical direction, both copper particles would suffer from the attractive force of iron particles, thus resulting in improved sintering neck growth between the two copper particles. A region in the sample containing $\mathrm{Cu}-\mathrm{Cu}$ necks around iron particles is extracted and shown in Figure 7. Iron particles surround the sintering necks in the red panes, whereas no iron particle was found around the region in the circle. The sintering necks in the red panes had already formed. No neck was generated in the circle in the 17 th $\mathrm{min}$. In the 27 th $\mathrm{min}$, the sintering necks grew obviously larger than that in the circle.

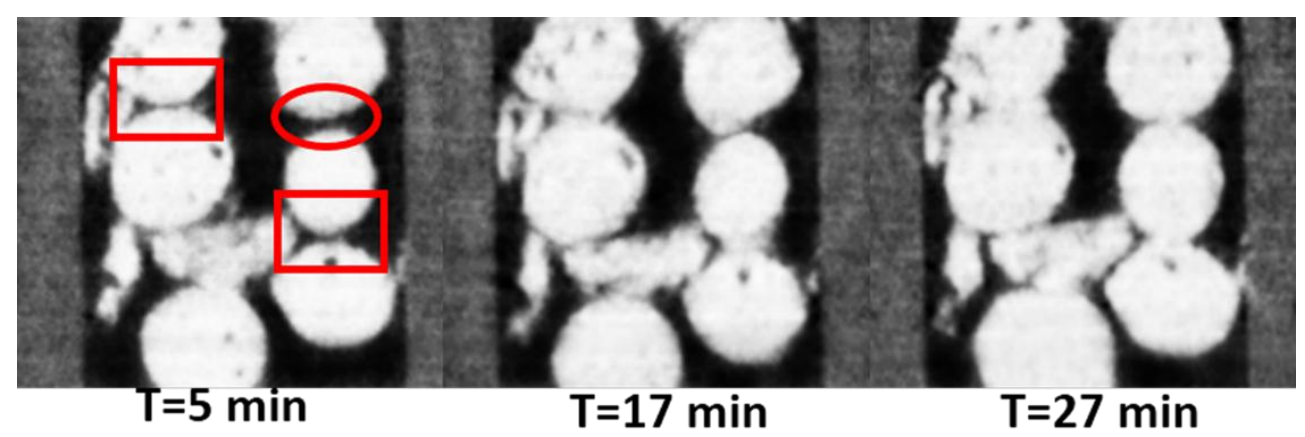

Figure 7. Evolution of $\mathrm{Cu}-\mathrm{Cu}$ necks around iron particles in vertical direction at different sintering times. 
Scanning electron microscopy (SEM, model XL30 ESEM-TMP, Philips, Amsterdam, The Netherlands) was also performed to verify the SR-CT images. The sample we used for SR-CT was powder-piled in a tiny quartz capillary. Extracting the sample completely is difficult when the capillary is broken. Hence, another sample that is relatively larger is prepared for the SEM experiments. The larger sample was sintered under the same condition and parameters. Similar phenomena can also be observed from SEM images of material section. In Figure 8, the anisotropic neck growth can be clearly observed. The sintering neck growth in the vertical direction marked with red panes is significantly faster than those in the other direction marked with blue panes. The SEM images of material sections agree well with the internal microstructure of SR-CT results.

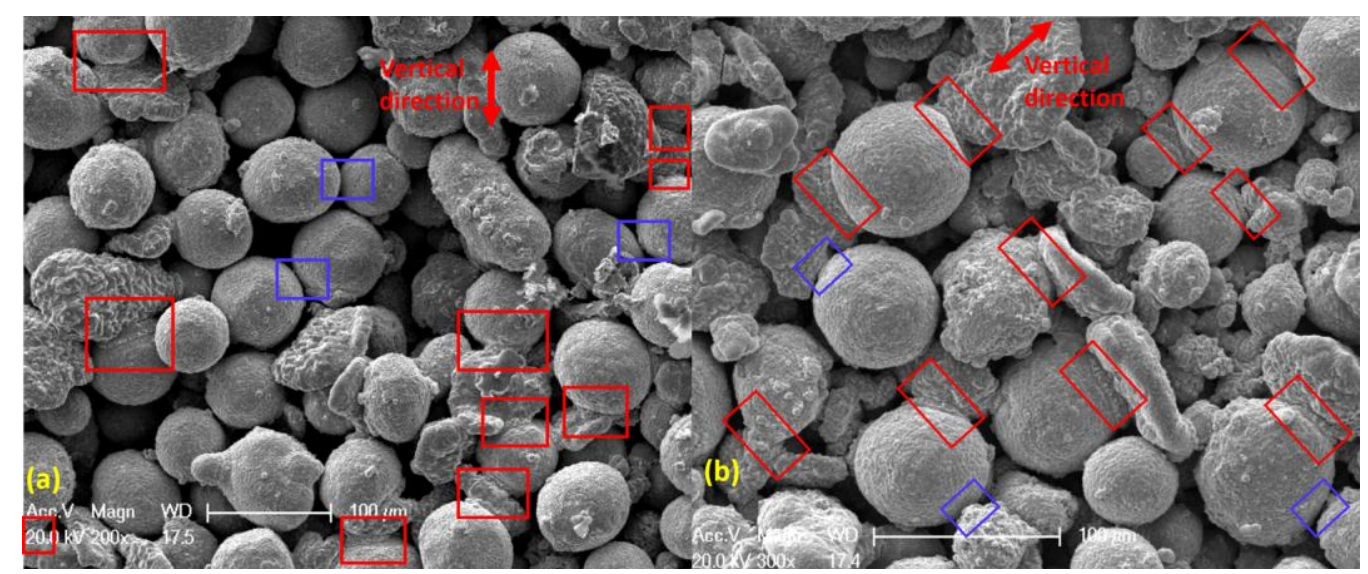

Figure 8. SEM images of two different regions of material in (a) and (b) respectively.

The curve of the sintering neck area of the sample is extracted to analyze the influence of the magnetic force between iron and copper micro particles. A watershed algorithm was used to obtain the morphological parameters [19]. The sintering neck between two particles can be distinguished and the size of sintering neck can be counted. The result is shown in Figure 9. The red line stands for the average projecting size of sintering necks in the vertical direction, and the black line stands for the average projecting size of sintering necks in the horizontal direction. The blue line showed the vertical sintering neck growth in a selected local region with several small iron particles to avoid iron particle influence. In the early stage, the three curves showed a similar slope, which indicated that the sintering neck growth rates are similar because of the slow evolution at a low temperature. Second, in the intermediate period, the slopes became different and the growth rates in the vertical direction shown by red and blue were both faster than that in the horizontal direction. This phenomenon is caused by gravity and possible magnetic force. Third, the slope of the red curve was larger than the slope of the blue curve, indicating that the region with iron particles had a faster growth rate than the region with no iron particles. This phenomenon can be clearly attributed to the influence of magnetic force between iron and copper particles rather than gravity. In the final stage of sintering, sintering neck growth would slow down and when the temperature is higher than the Curie point of iron, the magnetic susceptibility of iron became much lower. The magnetic force can be ignored at this high temperature. Hence, the neck growth rates in different directions became similar again. 


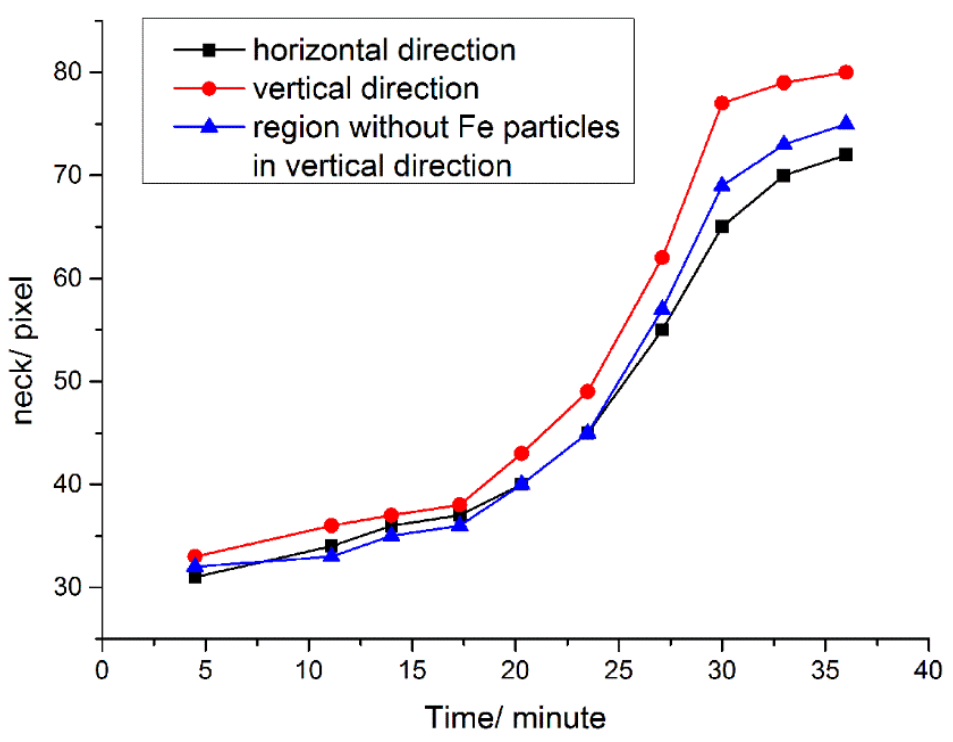

Figure 9. Sintering neck growth curves in different directions in different regions.

\subsection{Effect Parameters of the Magnetic Force Influence}

First, the magnitude of magnetic force of a magnetic pole is given as $\vec{F}=(\vec{m} \times \vec{B})$, where $\vec{m}$ is the vector sum of magnetic dipole moment of the particle. Hence, $\vec{m}=V \times \vec{M}, \vec{M}$ is magnetic intensity that represents the vector sum of molecular magnetic moment in per unit volume, and $V$ stands for volume [20]. The magnetic force between particles is proportional to particle volume, the magnetic force would be stronger around bigger iron particles, and the improvement or restraint would also be stronger. To verify this speculation, we extracted a series of regions containing iron particles of different volume. All the iron and copper particles were arranged in the horizontal direction. Figure 10a shows no iron particles; hence, the sintering neck formed the earliest without repulsive force of the iron particle. In Figure 10b,c, the volume of iron particles became increasingly larger, and the sintering necks around the iron particles subsequently formed. In Figure 10b, a small iron particle is found near the two copper particles, and the sintering neck formed at the 23th min, while the sintering neck formed at the 27th min in Figure 10c was surrounded by a large iron particle.

These three regions were quantitatively analyzed. Figure 11 shows the different sintering neck growth curves in these regions. In region a without iron particles, the sintering neck growth accelerated early at about the 14th min and quickly reached the final sintering stage at the 23rd min. In region c with a large iron particle, the sintering neck growth was slow before the 27th min because of the restraint caused by magnetic force. When the temperature increased beyond the Curie point of iron and the magnetic force disappeared, the neck growth became extremely fast. In Figure 10b, the volume of iron particles is intermediate between regions a and c. Hence, the neck growth curve also fell between them.

Second, particle shape possibly influenced the magnetic force between particles when the demagnetizing field was considered. A demagnetization field was observed when a specimen was magnetized by an external magnetic field. The demagnetization field can be expressed as $\vec{H}_{d}=-N \times \vec{M}$, where $N$ stands for the demagnetization factor, which is related to shape. $N$ is large when a spindly particle is magnetized through the short axis direction; conversely, $N$ is small when the particle is magnetized through the long axis direction [21]. Hence, the magnetic force between the tip of the iron particle and the copper particle would be stronger while the magnetic force between the side edges of iron and copper particles would be smaller. Two local regions are further discussed. Figure 12a shows the local region in Figure 10c more clearly. The $\mathrm{Cu}-\mathrm{Cu}$ sintering necks in the red and blue panes were near the side edge and tip of an iron particle, respectively. The sintering 
neck in the red pane grew obviously larger than that in the blue pane at the same sintering time. Similarly in Figure 12b, Fe-Cu sintering necks were found at the side edge and the tip of an iron particle. The sintering neck in the red pane near the side edge of the iron particles was larger than that in the blue pane near the tip of iron particles. Table 1 shows the neck growth and curvature of the corresponding parts of the iron particles. The sintering neck growth near sharp iron particles is larger than those near obtuse iron particles in the horizontal direction. These experimental images agree well with the possible phenomena speculated from the demagnetization effect of micro metal particles in microwave sintering.
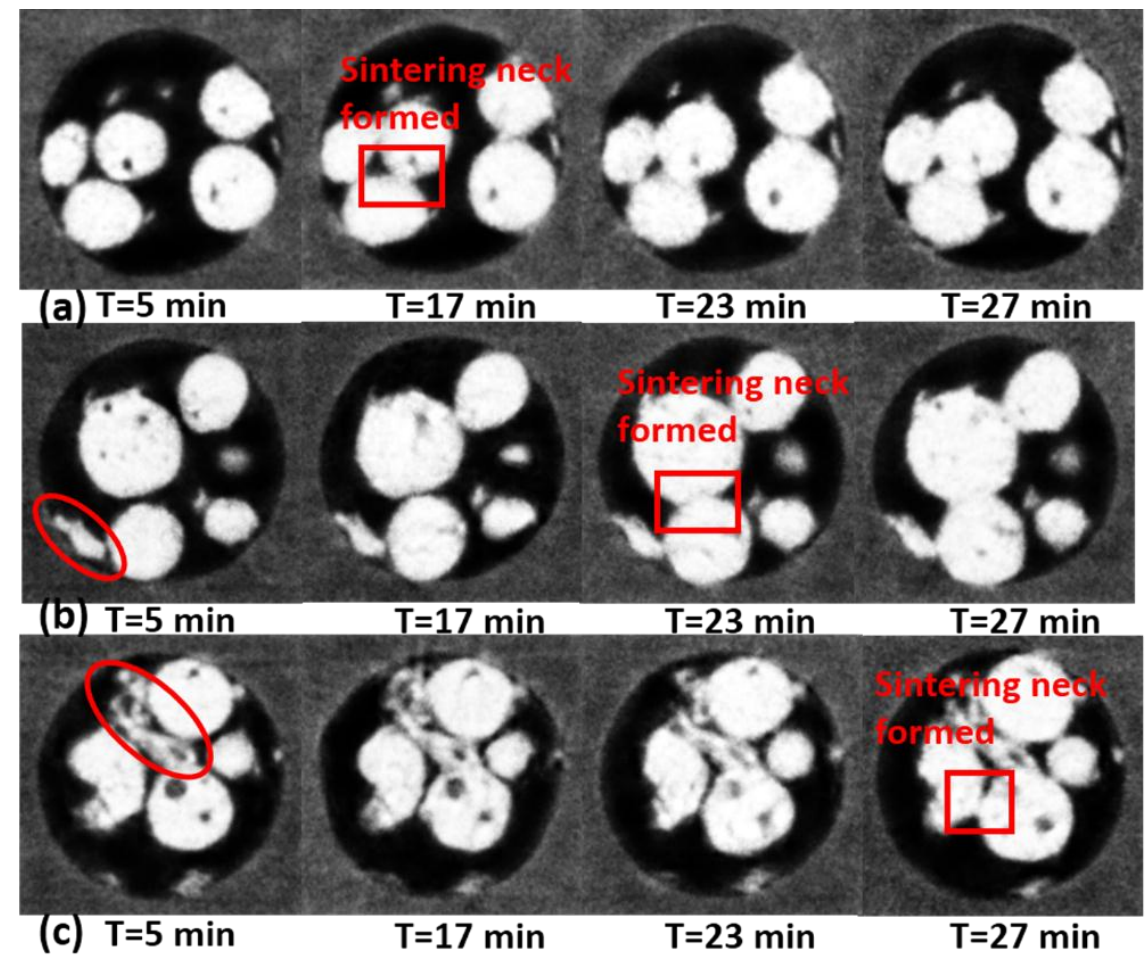

Figure 10. Microstructure evolution of $\mathrm{Cu}-\mathrm{Cu}$ necks around iron particles in the horizontal direction. (a) no iron particles; (b) a small iron particle; (c) a large iron particle.

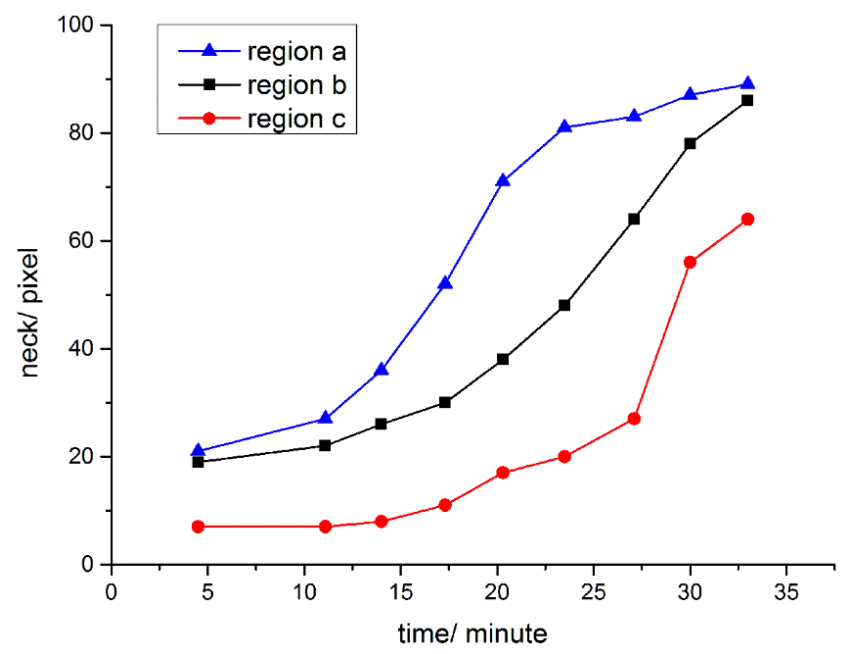

Figure 11. Sintering necks' growth curves of regions with different volume iron particles in the horizontal direction. 

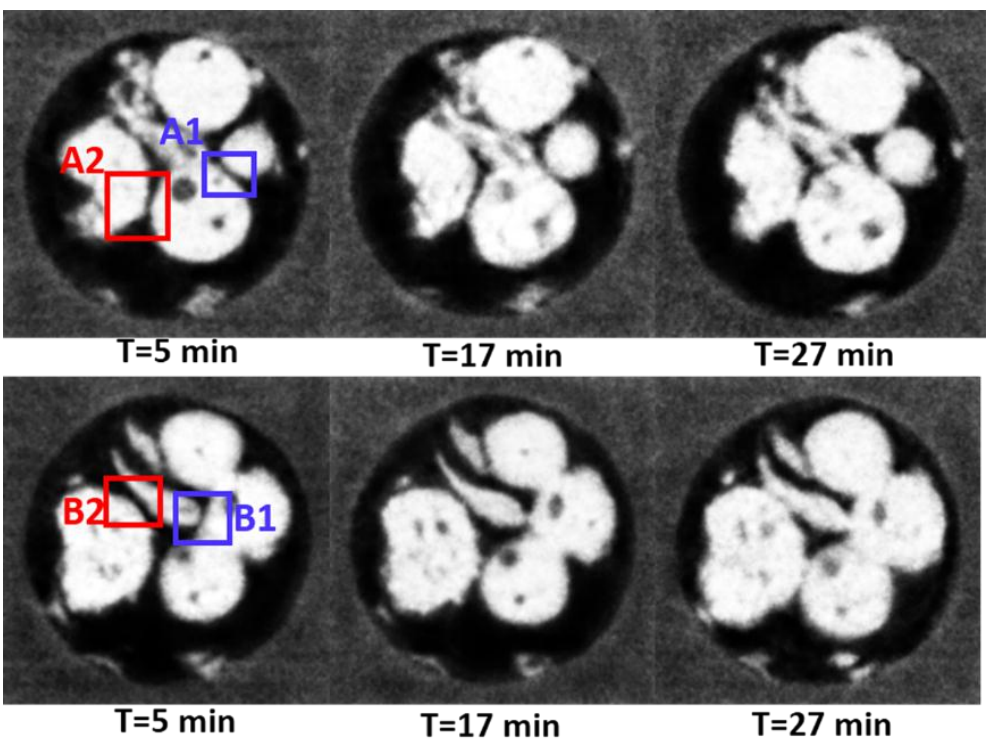

Figure 12. Particle shape's influence on sintering neck growth.

Table 1. Iron particles' curvature and neck growth in the local regions.

\begin{tabular}{ccc}
\hline Region & Curvature/Pixel & Neck Growth/Pixel \\
\hline A1 & 12 & 23 \\
A2 & 353 & 50 \\
B1 & 14 & 24 \\
B2 & 391 & 73 \\
\hline
\end{tabular}

\section{Conclusions}

The magnetic force between reversely magnetized micro metal particles in microwave sintering was investigated. SR-CT was applied to observe microstructure evolution in situ. Iron particles improve and restrain the sintering neck growth in vertical and horizontal directions, respectively. This special phenomenon confirmed that the attractive and repulsive forces between $\mathrm{Cu}$-Fe particles influenced the sintering neck growth. The kinetic curves were also consistent with the magnetic force model. On the basis of a series of microstructure evolution images, we confirmed that the volume of iron particles was proportional to the influence on sintering neck growth. The shape of iron particles also influenced the local magnetic force between iron particles. The influence of magnetic force might result in non-uniform densification in vertical and horizontal directions in microwave sintering. This study could be used as a reference for anisotropic material preparation.

Acknowledgments: This paper was supported by the National Nature Science Foundation of China (No. 11272305, No. 11172290, No. 11472265, No. 10902108), the National Basic Research Program of China (973 Program, No. 2012CB937504) and Anhui Provincial Natural Science Foundation (No. 1508085MA17).

Author Contributions: Yu Xiao and Feng Xu analyzed the experimental data and wrote the paper; Feng Xu and Xiaofang Hu conceived and designed the experiment; and Yu Xiao, Bo Dong and Wenchao Liu prepared the sample and performed the experiment.

Conflicts of Interest: The authors declare no conflict of interest.

\section{References}

1. Luo, S.D.; Yang, Y.F.; Schaffer, G.B.; Qian, M. Novel fabrication of titanium by pure microwave radiation of titanium hydride powder. Scr. Mater. 2013, 69, 69-72. [CrossRef]

2. Demirskyi, D.; Cheng, J.; Agrawal, D.; Ragulya, A. Densification and grain growth during microwave sintering of titanium diboride. Scr. Mater. 2013, 69, 610-613. [CrossRef] 
3. Thuault, A.; Savary, E.; Hornez, J.C.; Moreau, G.; Descamps, M.; Marinel, S.; Leriche, A. Improvement of the hydroxyapatite mechanical properties by direct microwave sintering in single mode cavity. J. Eur. Ceram. Soc. 2014, 34, 1865-1871. [CrossRef]

4. Upadhyaya, A.; Tiwari, S.K.; Mishra, P. Microwave sintering of W-Ni-Fe alloy. Scr. Mater. 2007, 56, 5-8. [CrossRef]

5. Roy, R.; Agrawal, D.; Cheng, J.; Gedevanishvili, S. Full sintering of powdered-metal bodies in a microwave field. Nature 1999, 399, 668-670.

6. Savary, E.; Marinel, S.; Gascoin, F.; Kinemuchi, Y.; Pansiot, J.; Retoux, R. Peculiar effects of microwave sintering on ZnO based varistors properties. J. Alloy. Compd. 2011, 509, 6163-6169. [CrossRef]

7. Rybakov, K.I.; Semenov, V.E.; Link, G.; Thumm, M. Preferred orientation of pores in ceramics under heating by a linearly polarized microwave field. J. Appl. Phys. 2007, 101, 084915. [CrossRef]

8. Janney, M.A.; Kimrey, H.D.; Allen, W.R.; Kiggans, J.O. Enhanced diffusion in sapphire during microwave heating. J. Mater. Sci. 1997, 32, 1347-1355. [CrossRef]

9. Asoka-Kumar, P.; O'brien, K.; Lynn, K.G.; Simpson, P.J.; Rodbell, K.P. Detection of current-induced vacancies in thin aluminum-copper lines using positrons. Appl. Phys. Lett. 1996, 68, 406-408. [CrossRef]

10. Xu, F.; Xiao, Y.; Hu, X.F.; Li, Y.C.; Liu, W.C.; Dong, B. In situ investigation of Al-Ti mixed metal system microwave sintering by synchrotron radiation computed tomography. J. Instrum. 2016, 11, C02074. [CrossRef]

11. Ahn, J.H.; Lee, J.N.; Kim, Y.C.; Ahn, B.T. Microwave-induced low-temperature crystallization of amorphous Si thin films. Curr. Appl. Phys. 2002, 2, 135-139. [CrossRef]

12. Birnboim, A.; Calame, J.P.; Carmel, Y. Microfocusing and polarization effects in spherical neck ceramic microstructures during microwave processing. J. Appl. Phys. 1999, 85, 478-482. [CrossRef]

13. Rybakov, K.I.; Olevsky, E.A.; Semenov, V.E. The microwave ponderomotive effect on ceramic sintering. Scr. Mater. 2012, 66, 1049-1052. [CrossRef]

14. Xu, F.; Dong, B.; Hu, X.F.; Wang, Y.; Liu, W.; Li, Y. Discussion on magnetic-induced polarization Ampere's force by in situ observing the special particle growth of alumina during microwave sintering. Ceram. Int. 2016, 42, 8296-8302. [CrossRef]

15. Badev, A.; Heuguet, R.; Marinel, S. Induced electromagnetic pressure during microwave sintering of $\mathrm{ZnO}$ in magnetic field. J. Eur. Ceram. Soc. 2013, 33, 1185-1194. [CrossRef]

16. Grupp, R.; Nöthe, M.; Kieback, B.; Banhart, J. Cooperative material transport during the early stage of sintering. Nat. Commun. 2011, 2, 298. [CrossRef] [PubMed]

17. Xu, F.; Li, Y.C.; Hu, X.F.; Niu, Y.; Zhao, J.; Zhang, Z. In situ investigation of metal's microwave sintering. Mater. Lett. 2012, 67, 162-164. [CrossRef]

18. Xu, F.; Dong, B.; Hu, X.; Xiao, Y.; Wang, Y. In situ investigation on rapid microstructure evolution in extreme complex environment by developing a new AFBP-TVM sparse tomography algorithm from original CS-XPCMT. Opt. Lasers Eng. 2016. [CrossRef]

19. Gonzalez, R.C.; Woods, R.E.; Eddins, S.L. Digital Image Processing Using Matlab; Publishing House of Electronics Industry: Beijing, China, 2005; pp. 315-317.

20. Edward, M.P.; David, J.M. Electricity and Magnetism, 3rd ed.; Cambridge University Press: New York, NY, USA, 2013; pp. 536-550.

21. Yan, M.; Peng, X.L. Magnetism Fundamental and Magnetic Materials; Zhejiang University Press: Hangzhou, China, 2006; pp. 7-8.

(C) 2017 by the authors; licensee MDPI, Basel, Switzerland. This article is an open access article distributed under the terms and conditions of the Creative Commons Attribution (CC BY) license (http://creativecommons.org/licenses/by/4.0/). 